\title{
Elective Anesthesia and Surgery in Veteran Patients Abusing Cocaine - Factors Influencing Management Decisions
}

\author{
Sakawi $\mathrm{Y}^{*}$ and Whitley ML
}

\section{Department of Anesthesiology Service, Birmingham VA Medical Center, USA}

${ }^{*}$ Corresponding author: Sakawi Y, Department of Anesthesiology Service, Birmingham VA Medical Center, 700 South 19th Street, Birmingham AL 35233, USA, E-mail: yasakawi@uab.edu

Citation: Sakawi Y, Whitley ML (2015) Elective Anesthesia and Surgery in Veteran Patients Abusing

Cocaine - Factors Influencing Management Decisions. J Anesth Pati Care 1(1): 103. doi: 10.15744/24565490.1.103

Received Date: July 02, 2015 Accepted Date: September 29, 2015 Published Date: October 01, 2015

\begin{abstract}
Background: Cocaine abuse remains to be a significant anesthesia challenge making decision to proceed with anesthesia in patients in general and veterans in particular testing positive for cocaine quite controversial.

Aim: Review of the current literature regarding the decision making process to proceed with anesthesia and surgery in cocaine positive patients.

Method: Literature was reviewed and views to support or oppose the decision to proceed with anesthesia were discussed.

Results: Currently, there is lack of scientific evidence that supports cancelling surgeries for veterans testing positive for cocaine by urine drug screen the morning of surgery.

Conclusions: It might be reasonable to proceed with elective surgeries in patients testing positive by urine drug screen for cocaine the morning of surgery. Consideration should be made for co-existing diseases and the extent and urgency of the surgical procedure. Overt clinical signs of cocaine toxicity would be a much better indicator of the need to postpone elective surgery.
\end{abstract}

KeyWords: Veterans; Cocaine; Management decisions

\section{Introduction}

Cocaine use in the United States has increased from an estimated 34 million Americans in 2004 to 36.8 million in 2008 . This corresponds to approximately $15 \%$ of Americans over the age of 12 years with use at least once in their life. Data from the National Survey on Drug Use and Health (2003-2006) indicate that 7.1\% of veterans meet criteria for a past year substance abuse disorder [1]. The 2008 National Survey on Drug Use and Health showed 14.1\% of respondents with military service history had used cocaine more than 12 months prior [2]. A chart review of 117 veterans over the age of 50 treated for crack cocaine found that nearly $15 \%$ began their cocaine use after the age of 50 [3]. The rising prevalence of drug use among middle-aged adults, in part reflecting aging of the baby boom generation [4], poses challenges for health care of middle-aged veterans.

Cocaine inhibits the reuptake of norepinephrine at the neuromuscular junction and results in sodium and potassium channel blockade [5,6]. This results in increased blood pressure, heart rate, body temperature and potential for coronary artery spasm and ischemia-induced cardiac arrhythmias [7]. Because cocaine increases levels of catecholamines [8], any patient who presents for surgery with cocaine in their system could theoretically have anesthesia complications. However, this risk has not been confirmed by prospective controlled studies, leaving preoperative decision making unguided by evidence.

Because of cocaine's cardiovascular effects, veterans with a history of cocaine abuse presenting for elective surgery are of particular concern to anesthesiologists and surgeons. However, while there is concern that recent or past use of cocaine may increase the risk of hemodynamic instability and adverse outcomes, there is very little data to guide clinical practice. As a result, there are no current guidelines and clinical practices vary widely. In a survey of anesthesia services at all Veteran Administration (VA) hospitals, service chiefs reported on group policies and practices for routine urine drug screening (UDS) for surgical patients and case cancellations [9]. Survey results showed perioperative management of cocaine abusing patients varies widely between institutions based mainly on the culture of the institution and individual anecdotes [9]. Confronted with a patient whose history includes cocaine abuse, some surgical teams proceed whenever the patient presents without acute intoxication, while others order UDS immediately prior to surgery and proceed only if the test shows no evidence of recent cocaine usage. The lack of clear guidelines is particularly vexing to practitioners faced with patients whose urine tests positive for recent usage but who exhibit no signs of acute intoxication. Those patients' procedures are routinely, but not universally cancelled. Given the lack of clinical evidence, it is not known whether these cancellations save the patients from an increased risk of adverse outcomes or needlessly waste VA resources through case cancelations and inconvenience to veterans. 


\section{Cocaine is a Significant Problem among Veterans}

The most recent estimates of cocaine abuse among veterans come from a 1999 VA study that reported $1.7 \%$ of veterans have an ICD-9 code for a cocaine-related diagnosis including a diagnosis of cocaine abuse or cocaine dependence. Veterans typically receive this code when they get treated at a VA facility for cocaine related drug use, and thus this is likely an underestimate of the true prevalence. The VA population for fiscal year 2010 was estimated to be 5.2 million [10]. Applying the $1.7 \%$ rate of cocaine abuse in the VA population, an estimated 88,400 current VA service recipients have a cocaine use disorder severe enough to merit the ICD-9 diagnosis of cocaine abuse. It can be assumed that a higher percentage of veterans abuse cocaine but are not identified with ICD-9 codes.

The 2008 National Survey on Drug Use and Health reported that $0.9 \%$ of respondents who had previous military service had used cocaine within the last year, with over half of those veterans having used it within 30 days, and $14.1 \%$ had used cocaine more than 12 months ago. Based on an estimated 5.2 million patients in the VA care system, this corresponds to 46,800 veterans who have used cocaine within the last year, and 733,200 who have used cocaine over 12 months ago. This estimate of cocaine abuse in veterans is most likely also an underestimation because survey participation can be non-representative and results rely on selfreporting.

Abusing cocaine is usually accompanied by abusing multiple other drugs. There is a high incidence of serious psychological distress (SPD) and substance abuse disorder among veterans as illustrated in Figure 1 [1]. It is likely that the more general diagnosis of substance abuse includes patients with cocaine abuse. Substance abuse is more prevalent in impoverished and homeless populations, and veterans have a higher rate of poverty and homelessness suggesting that the rate of cocaine abuse may be significant in the veteran population.

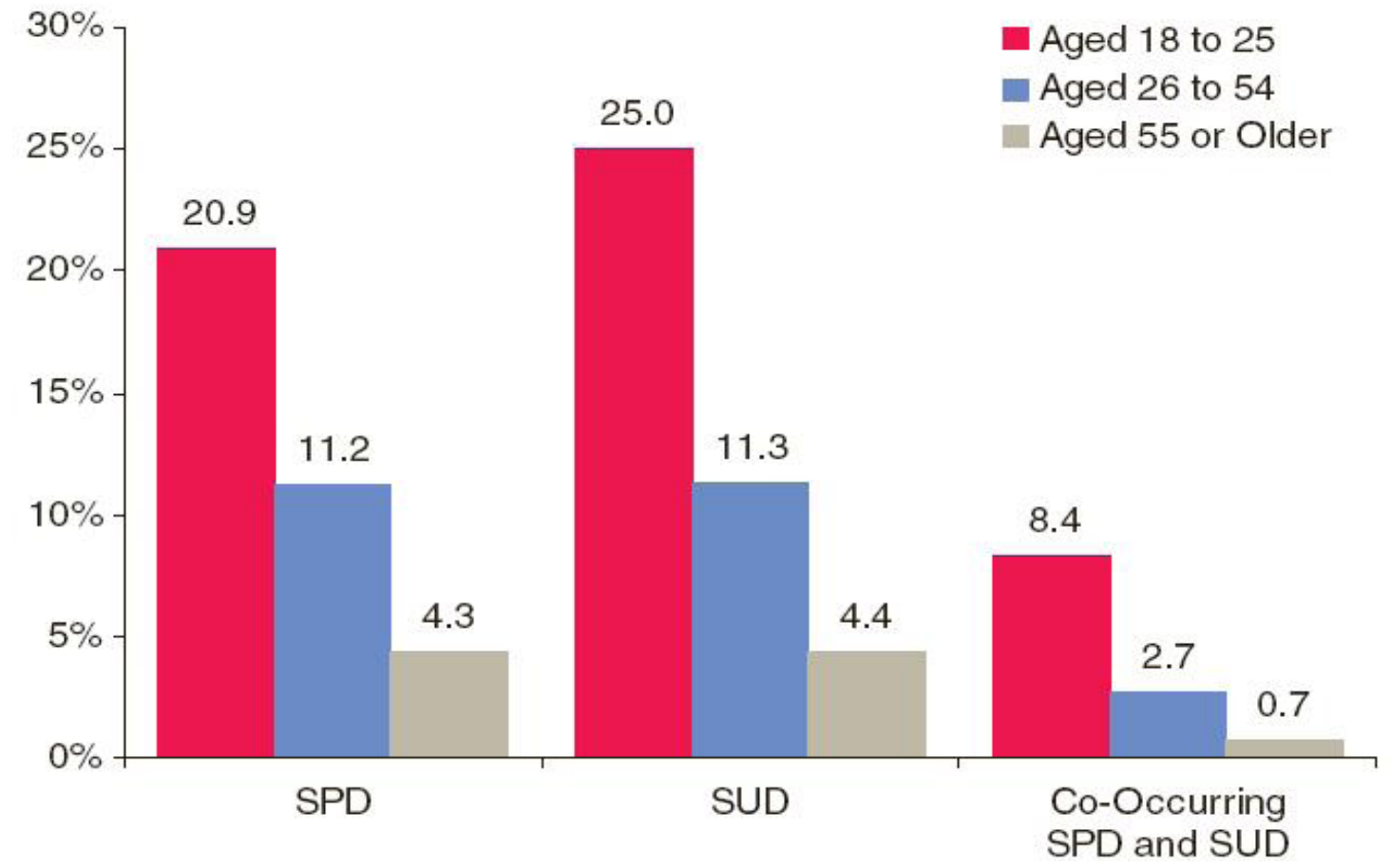

Figure 1: Prevalence of Serious Psychological Distress (SPD), Substance Use Disorder (SUD), and Co-Occurring SPD and SUD in the Past Year among Veterans, by Age 2004-2006

\section{Chemical Structure, Pharmacokinetics and Pharmacodynamic of Cocaine}

In order to understand the influence of cocaine on the human body and it's detection by drug testing, it is necessary to understand the pharmacology of cocaine. Cocaine or benzoylmethylecognine is an ester of benzoic acid and methylecognine. The salt form is freely soluble in water, which allows it to be injected, but its lipid solubility also enables it to be absorbed through the nasal mucosa [11]. In contrast, cocaine alkaloid, also known as freebase or crack cocaine, is a colorless, odorless, crystalline substance, insoluble in water but soluble in ether, acetone or alcohol and typically smoked [12].

Three routes of administration are commonly used for cocaine: snorting, smoking and injecting and the onset and duration of effects varies by route of administration. Cocaine has a relatively short half-life in plasma ranging from 30-90 minutes [12]. It is rapidly cleared from plasma by esterases, of which the most important is plasma cholinesterase. Eighty to $90 \%$ of cocaine is metabolized to form ecgonine methyl ester and benzoylecgonine. The methyl esters are formed by rapid enzymatic hydrolysis by plasma and liver esterases and have a half-life of 4 hours. Benzoylecgonine is formed mainly by spontaneous non-enzymatic hydrolysis. It has a half-life of about 6-7.5 hours. Ten to $20 \%$ of cocaine undergoes n-demethylation in the liver to nor-cocaine, a cocaine metabolite with considerable pharmacologic activity [11]. Approximately 1-5\% of cocaine is not metabolized and undergoes urinary clearance. 
Because the half-lives of ecgonine methyl ester and benzoylecgonine are much longer than cocaine, they can be detected in the urine for up to 10 days. The metabolites possess no cocaine-like stimulation effects on the cardiovascular system but provide the basis for urine drug testing.

Cocaine blocks reuptake of catecholamines into the presynaptic nerve terminals in both central and peripheral central nervous systems causing the accumulation of catecholamines in synaptic clefts. This results in increased $\alpha$-adrenoreceptor, $\beta$-adrenoreceptor and dopamine receptors stimulation via indirect sympathomimetic action. Alpha and beta receptor stimulation results in vasoconstriction, cardiac stimulation and gastrointestinal effects. Cocaine shifts the dose response curve to norepinephrine to the left and enhances its maximal effects [8]. Cocaine is also a local anesthetic and competitively blocks the fast voltage-sensitive sodium $\left(\mathrm{Na}^{+}\right)$channels of nerve cells, resulting in slowing of conduction of action potential.

\section{Effects of Cocaine on Different Systems in Humans}

The effects of cocaine on the central nervous system (CNS) depend on the amount of drug taken, the user's past drug experience, route of administration and concomitant utilization of other drugs. The amount of cocaine utilized range from a small $100 \mathrm{mg}$ dose to as high as several hundred milligrams. Amounts as high as 10 grams have been reportedly taken. The effects start with a sense of euphoria and self-confidence and may progress to restlessness, extreme anxiety, vertigo, muscle twitching, paranoia, convulsions and coma. There is a suppression of the need to eat or sleep [13].

Cerebral ischemia, infarction, hemorrhagic strokes and seizures have been reported with cocaine use [8]. Barash, et al. conducted a study with limited number of patients and found that use of intranasal cocaine did not result in any significant changes in blood pressure, pulse, cardiac index, left ventricular stroke index, peripheral vascular resistance, or pulmonary resistance [14]. However, the results of this study do not take into account respiratory, renal, hematologic, or neurologic deleterious effects of cocaine such as increased platelet aggregability leading to in situ thrombus formation [15].

Cardiac complications with both acute and chronic cocaine use have been reported. Cocaine increases myocardial oxygen demand while reducing its supply [16]. This results in myocardial infarction and ischemia [15], cardiac dysrhythmias, dilated cardiomyopathy and sudden death. Acute intranasal cocaine administration results in coronary vasospasm seen during cardiac catheterization [7].

Pulmonary complications related to cocaine use include airway injury by the products of combustion of cocaine or from the ignition of the highly volatile ethers used in the extraction process of the drug. This may be severe enough to cause tracheal stenosis [17]. Cocaine use can exacerbate asthma, cause pulmonary edema, pulmonary hemorrhage or infarction and overall deterioration of pulmonary function [18].

Cocaine can cause accelerated hypertension and renal failure [19]. Cocaine use during pregnancy is an independent contributor to the risk of placental abruption, preterm labor, precipitate delivery and stillbirth [20].

\section{Lack of Knowledge about the Interaction between Anesthesia and Cocaine}

Animal Models: Animal studies suggest potential for an unfavorable interaction between cocaine and anesthesia. Results from anesthetized cats suggest potentiation of cardiac responses to adrenergic stimuli possibly due to the presynaptic block of reuptake of norepinephrine [21].

Koehntop, et al. studied the effects of cocaine on the ability of epinephrine to induce arrhythmias during halothane-nitrous oxide anesthesia in dogs [22]. The results of the study suggest that cocaine interferes with intra-neuronal uptake of neuromediators leading to a stepwise increase in the arrhythmogenicity of epinephrine. Other studies in different animal models showed similar effects [23-26].

About $2 \%$ of pregnancy-related DVT develops in the upper limb [25]. But the cases of DVT developing in association with reproductive technologies occur predominantly in the upper limb or neck in pregnant patients who had ovarian hyperstimulation syndrome [17].

Human Studies: Unfortunately, the interaction between anesthesia and cocaine in humans is less well understood and more complicated than animal models would suggest. Animal models permit simulation of acute cocaine intoxication by reliably administering the drug at a known time followed by measuring concrete outcomes. In humans, such studies are rarely possible.

Because medical grade cocaine is sometimes used as a local anesthetic and vasoconstrictor for nasal procedures, its effects were studied in a small number of patients undergoing coronary artery surgery who received topical cocaine to the nasal mucosa prior to nasal intubation. Eleven patients received cocaine hydrochloride as a $10 \%$ solution ( $1.5 \mathrm{mg} / \mathrm{kg})$ applied topically to the nasal mucosa before nasotracheal intubation. Seven patients had a similar procedure without cocaine. Blood pressure, pulse rate, cardiac index, left ventricular stroke work index, total peripheral vascular resistance and pulmonary vascular resistance were measured. There were no important differences in cardiovascular function between patient groups. The rapid rise in plasma cocaine concentration, reaching $331 \mathrm{ng} / \mathrm{mL}$ at 30 minutes and $320 \mathrm{ng} / \mathrm{mL}$ at 45 minutes, bore no apparent relationship to any changes in cardiovascular function. The study concluded that under the conditions of this study, administration of topical cocaine does not exert any clinically significant sympathomimetic effect and appears to be well tolerated in anesthetized patients with coronary artery disease [14]. The limitations to this study include the small sample size and the inability to assess if the obtained plasma cocaine levels correspond to levels obtained from illicit use. 
Mittleman, et al. interviewed 3946 patients with acute myocardial infarction due to cocaine, and found that the risk of myocardial infarction onset was elevated 23.7 times over baseline in the 60 minutes after cocaine use. They concluded that cocaine use is associated with a large abrupt and transient increase in the risk of acute myocardial infarction in patients who are otherwise at relatively low risk [15]. Anesthetic agents and surgical stress can depress cardiac function which may be compounded by the effects of cocaine, but there are no prospective controlled studies examining this interaction.

A review of the literature located only one prospective study investigating the safety of anesthesia in patients with a positive UDS the day of surgery [27]. This study compared healthy patients (defined as classes 1 and 2 by the American Society of Anesthesiologists "ASA" health classification system) who presented for surgery with a UDS positive for cocaine the day of surgery to patients with a negative UDS for cocaine who were undergoing routine outpatient surgical procedures. The study excluded patients with clinical signs of cocaine intoxication as defined by systolic, diastolic or mean non-invasive blood pressure and heart rate greater than or less than $10 \%$ of the baseline preoperative reading taken prior to the day of surgery, a heart-rate-corrected QT interval of greater than $500 \mathrm{~ms}$, or hyperthermia. The study found no increased intra-operative hemodynamic instability as defined by areater than $30 \%$ increase or decrease in heart rate compared to the preoperative value, or a greater than $40 \%$ increase or decrease in the mean arterial pressure compared to the preoperative value. No cardiac instability was reported, as defined by ST depression or the onset of new arrhythmias. Although the sample size was small (40 patients per group), the study concluded that general anesthesia could be safely administered in this patient population.

Another retrospective study examined the surgical outcomes in patients with positive drug screens for cocaine undergoing emergency trauma surgery [28]. Of 3,477 patients, 13\% (465 patients) tested positive for cocaine. Outcomes were similar for mortality ( $3 \%$ vs. $4 \%$ ), for the development of infections (18\% vs. $19 \%)$, neurologic complications $(2 \%$ vs. $1 \%)$ and median length of stay between cocaine positive and negative patient groups ( $p>0.5$ for all outcome measures. The study concluded that outcomes of patients undergoing trauma surgery are not negatively affected by the presence of positive urine cocaine toxicology. The study was retrospective, limited to trauma patients and all patients were under the age of 48 years. No analysis of preexisting health conditions or anesthesia management was made. Although the authors conclude that postponement of surgery is not indicated, they did not study outcomes in patients with positive cocaine urine toxicology in which surgery was postponed.

Surgical outcomes were also retrospectively studied in cocaine positive and negative trauma patients by matching 985 cocaine positive patients to cocaine negative patients by age, gender, mechanism of injury and severity of injury [29]. Only 274 patients had surgery and the timing of surgery was not addressed. The study found no difference in mortality, length of ICU stay or length of hospital stay between the two groups.

Information on the interaction between anesthesia and cocaine in humans is limited to retrospective outcome studies on trauma patients and a few small prospective studies.

\section{Cocaine Abusing Veterans may Have Higher Risks for Surgical Complications}

The existing studies on the effects of cocaine on anesthesia administration are limited to younger healthier patients than the veteran population [27]. The conclusion from Hill, et al [27] lacks external validity as applicable to older patients with more substantial medical comorbidities and to those patients undergoing more invasive non-ambulatory elective procedures. The same critique could be applied to other studies quoted in the trauma literature examining the impact of cocaine use upon surgical outcomes, in addition the cited patient population was predominantly male with ages between 34-55 years of age 29 . Analysis of 3.4 million veteran patients in 1999 showed that $72 \%$ of veteran patients had one or more chronic conditions on a list of 29 chronic medical problems. Thirty five percent had 3 or more chronic conditions. The study concluded that VA health care users have more chronic diseases than the general population [30].

A large cross-sectional study among outpatients examined the health status of elderly veterans stratified by age group compared with a nonveteran population [31]. Among 887,775 veterans who returned the survey (63.1\% of those surveyed), 663,729 ( $74 \%)$ were 65 years and older. Patient demographics, co-morbid conditions, and health status were assessed using the Veterans 36 -item short form (SF-36), a reliable and valid measure of health-related quality of life. Compared to nonveteran patients, elderly veteran enrollees had poorer health status and significantly lower health related quality of life [32].

Another study compared health status and disease profiles of ambulatory patients in VA and civilian healthcare settings [33]. A random sample of 2425 male veterans seeking care at four Boston-area VA outpatient clinics were compared to 1318 male seeking civilian outpatient care in three major metropolitan areas. The study illustrated that outpatients in the 4 VA clinics had more than twice the illness burden than their civilian patient counterparts. The chart review study of 114 veterans that showed that $14 \%$ of these patients began their abuse of cocaine after age 50 also documented coexisting diseases in this population. Vascular disease was present in $70.9 \%$ and hepatitis in $40 \%$.

As discussed previously, cocaine abuse among veterans is not limited to younger, healthier patients. It is highly likely that an Anesthesiologist may be required to make management decisions relating to cocaine abuse in older patients with one or more coexisting diseases that have their own anesthetic risks. There are no studies to address the safety of elective anesthesia for such patients and given the lack of clinical data, the anesthesiologist may be reluctant to provide anesthesia for elective procedures for patients with coexisting diseases in light of a positive UDC or admission of recent cocaine use. 


\section{Lack of Information to make Sound Evidence Based Clinical Decisions}

Anesthesiologists and surgeons are not guided by well-designed prospective studies that investigate the interaction between anesthesia and cocaine in the patients undergoing elective surgery. There are no clinical studies on the influence of cocaine abuse by patients with several co-existing diseases such as seen in the aging veteran population. This results in a lack of consensus among anesthesia providers and a wide variety of policies and practices. Citing fear of intraoperative cardiac arrhythmias such as ventricular tachycardia, frequent premature ventricular beats or torsades de pointes, some anesthesiologists routinely delay surgery for such patients until they obtain urine screen results and then cancel the case if the test is positive for cocaine [34]. Other anesthesiologists rely only on clinical signs and symptoms of acute cocaine intoxication and do not order any UDS the day of surgery. The same variation of practice exists among surgeons. Some surgeons take into consideration the results of a UDS for cocaine only if the procedure is totally elective and if delaying it would not negatively impact patient outcome. For instance, a surgeon might feel comfortable cancelling an elective hernia repair but proceed with surgery to remove a rapidly growing tumor. A survey of VA anesthesia services among the nation illustrates this lack of consensus [9]. Unnecessary case cancellations waste operating room resources and inconvenience patients and their families. Delays may also influence patient compliance with treatment.

\section{Conclusion}

Because limited clinical studies have failed to show an increased risk of anesthetic complications in patients who are not acutely toxic but have evidence of cocaine use by UDS, it seems reasonable not to cancel surgeries for some veteran patients based solely on a positive UDS the day of surgery. Consideration should be made of coexisting diseases and the extent and urgency of the surgical procedure. Surgeons and anesthesiologists may be reluctant to proceed if vascular stability is crucial or vasospasm would compromise the surgery since hemodynamic swings and vasospasm are theoretical risks of recent cocaine use. Patients undergoing procedures in which large blood loss is possible might likewise be postponed. The current evidence suggests that it might be reasonable to proceed with elective surgeries in patients testing positive by UDS for cocaine the morning of surgery especially if there is no significant co-morbidities. Overt clinical signs of cocaine toxicity would be a much better indicator of the need to postpone elective surgery. Formal guidelines for the perioperative management of cocaine-abusing patients would be helpful. Clinical outcome research is needed to provide clinicians with decision tools and evidence-based guidelines to guide management of veterans testing positive by UDS the day of surgery [9]. The tool becomes even more needed for veterans with multiple co-existing diseases undergoing complex procedures.

\section{References}

1. The Office of Applied Studies SAaMHSAS: Serious Psychological Distress and Substance Use Disorder among Veterans (2007) The National Survey on Drug Use and Health.

2. Studies USDoHaHSSAaMHSAOoA: National Survey on Drug Use and Health (2008) Ann Arbor, MI: Inter-university Consortium for Political and Social Research.

3. Busko M (2008) Study of Older Veterans Sheds Light on Cocaine Use. Medscape Medical News.

4. Colliver JD, Compton WM, Gfroerer JC, Condon T (2006) Projecting drug use among aging baby boomers in 2020. Ann Epidemiol 16: 257-65.

5. Van Dyke C, Barash PG, Jatlow P, Byck R (1976) Cocaine: plasma concentrations after intranasal application in man. Science 191: 859-61.

6. Van Dyke C, Jatlow P, Ungerer J, Barash PG, Byck R (1978) Oral cocaine: plasma concentrations and central effects. Science 200: 211-3.

7. Brogan WC 3rd, Lange RA, Glamann DB, Hillis LD (1992) Recurrent coronary vasoconstriction caused by intranasal cocaine: possible role for metabolites. Ann Intern Med 116: 556-61.

8. Brownlow HA, Pappachan J (2002) Pathophysiology of cocaine abuse. Eur J Anaesthesiol 19: 395-414.

9. Elkassabany N, Speck RM, Oslin D, Hawn M, Chaichana K, et al. (2013) Preoperative screening and case cancellation in cocaine-abusing veterans scheduled for elective surgery. Anesthesiol Res Pract doi.org/10.1155/2013/149892.

10. Office CB (2007) The Health Care System for Veterans: An Interim Report.

11. Warner EA (1993) Cocaine abuse. Ann Intern Med 119: 226-35.

12. Prakash A, Das G (1993) Cocaine and the nervous system. Int J Clin Pharmacol Ther Toxicol 31: 575-81.

13. Hammer RP Jr, Egilmez Y, Emmett-Oglesby MW (1997) Neural mechanisms of tolerance to the effects of cocaine. Behav Brain Res 84: $225-39$.

14. Barash PG, Kopriva CJ, Langou R, VanDyke C, Jatlow P, et al. (1980) Is cocaine a sympathetic stimulant during general anesthesia? JAMA $243: 1437-9$.

15. Mittleman MA, Mintzer D, Maclure M, Tofler GH, Sherwood JB, et al. (1999) Triggering of myocardial infarction by cocaine. Circulation 99: $2737-41$.

16. Boehrer JD, Moliterno DJ, Willard JE, Snyder RW 2 $2^{\text {nd }}$, Horton RP, et al. (1992) Hemodynamic effects of intranasal cocaine in humans. J Am Coll Cardiol 20: 90-3.

17. Taylor RF, Bernard GR (1989) Airway complications from free-basing cocaine. Chest 95: 476-7.

18. Weiss RD, Goldenheim PD, Mirin SM, Hales CA, Mendelson JH (1981) Pulmonary dysfunction in cocaine smokers. Am J Psychiatry 138: 1110-2.

19. Thakur V, Godley C, Weed S, Cook ME, Hoffman E (1996) Case reports: cocaine-associated accelerated hypertension and renal failure. Am J Med Sci 312: 295-8.

20. Kain ZN, Rimar S, Barash PG (1993) Cocaine abuse in the parturient and effects on the fetus and neonate. Anesth Analg 77: 835-45. 
21. Sabra R, Khoury HA, Bechara G, Sharaf LH, El-Bizri NM (2000) Post-junctional mechanisms involved in the potentiation of cardiac adrenergic responses by cocaine. Eur J Pharmacol 397: 139-50.

22. Koehntop DE, Liao JC, Van Bergen FH (1977) Effects of pharmacologic alterations of adrenergic mechanisms by cocaine, tropolone, aminophylline, and ketamine on epinephrine-induced arrhythmias during halothane-nitrous oxide anesthesia. Anesthesiology 46: 83-93.

23. Ritz MC, Cone EJ, Kuhar MJ (1990) Cocaine inhibition of ligand binding at dopamine, norepinephrine and serotonin transporters: a structure-activity study. Life Sci 46: 635-45.

24. Rockhold RW, Byrne M, Sprabery S, Bennett JG (1994) Urethane anesthesia reverses the protective effect of noncompetitive NMDA receptor antagonists against cocaine intoxication. Life Sci 54: 321-30.

25. Siegal N, Dow-Edwards D (2009) Isoflurane anesthesia interferes with the expression of cocaine-induced sensitization in female rats. Neurosci Lett 464: 52-6.

26. Tsukada H, Nishiyama S, Kakiuchi T, Ohba H, Sato K, et al. (1999) Isoflurane anesthesia enhances the inhibitory effects of cocaine and GBR12909 on dopamine transporter: PET studies in combination with microdialysis in the monkey brain. Brain Res 849: 85-96.

27. Hill GE, Ogunnaike BO, Johnson ER (2006) General anaesthesia for the cocaine abusing patient. Is it safe? Br J Anaesth 97: 654-7.

28. Ryb GE, Cooper C (2008) Outcomes of cocaine-positive trauma patients undergoing surgery on the first day after admission. J Trauma 65: 809-12.

29. Hadjizacharia P, Green DJ, Plurad D, Chan LS, Law J, et al. (2009) Cocaine use in trauma: effect on injuries and outcomes. J Trauma 66: 491-4.

30. Yu W, Ravelo A, Wagner TH, Phibbs CS, Bhandari A S, et al. (2003) Prevalence and costs of chronic conditions in the VA health care system. Med Care Res Rev 60: 146-67.

31. Selim AJ, Berlowitz DR, Fincke G, Cong Z, Rogers W, et al. (2004) The health status of elderly veteran enrollees in the Veterans Health Administration. J Am Geriatr Soc 52: 1271-6.

32. Kazis LE, Miller DR, Clark J, Skinner K, Lee A, et al. (1998) Health-related quality of life in patients served by the Department of Veterans Affairs: results from the Veterans Health Study. Arch Intern Med 158: 626-32.

33. Rogers WH, Kazis LE, Miller DR, Skinner KM, Clark JA, et al. (2004) Comparing the health status of VA and non-VA ambulatory patients: the veterans' health and medical outcomes studies. J Ambul Care Manage 27: 249-62.

34. Bauman JL, Grawe JJ, Winecoff AP, Hariman RJ (1994) Cocaine-related sudden cardiac death: a hypothesis correlating basic science and clinical observations. J Clin Pharmacol 34: 902-11. 